\title{
CONOCIENDO Y VALORANDO NUESTROS RECURSOS AMAZÓNICOS A TRAVÉS DE LAS TECNOLOGÍAS DE LA INFORMACIÓN Y COMUNICACIÓN (TIC), UN ESTUDIO DE CASO: Arapaima gigas
}

\author{
Melba del Rocío CORREA-TANG ${ }^{1}$, José Lisbinio CRUZ GUIMARAES ${ }^{2}$, Saúl Alexander PINEDO FLOR ${ }^{3}$, \\ Kember Mateo MEJÍA CARHUANCA, Mónica Paola GARCÍA CóRDOVA ${ }^{4}$ \\ 1 Instituto de Investigaciones de la Amazonía Peruana (IIAP), Programa de Investigación en Biodiversidad Amazónica (PIBA). \\ Iquitos, Perú, rcorrea@ iiap.org.pe; kmejia@ iiap.org.pe \\ 2 Colegio Nuestra Señora de la Salud, Iquitos, Perú, josecruzguimaraes@ gmail.com \\ 3 Profesor de Informática, Iquitos, Perú, saul920@ gmail.com \\ 4 Universidad Nacional de la Amazonía Peruana - Estudiante de la Facultad de Agronomía, Escuela de Gestión Ambiental.
}

\section{RESUMEN}

En muchas instituciones educativas de Loreto y del país, la carencia de medios y materiales, y la ausencia de prácticas de diseño, producción y uso de recursos educativos, con base a las necesidades de los estudiantes, limitan el mejoramiento de la calidad educativa. Ante esta realidad es necesario propiciar el diseño, la producción y el uso de recursos educativos, cumpliendo un rol preponderante las Tecnologías de la Información y Comunicación (TIC), lo que en el futuro permitirá mejorar los procesos de enseñanza-aprendizaje. En concordancia con lo citado se elaboró el material interactivo "El Gigante del Amazonas: paiche Arapaima gigas", con el objetivo de contribuir a innovar el currículo educativo y la consolidación de los valores de conservación y manejo de los recursos amazónicos a edades tempranas. La información técnica fue traducida en un lenguaje sencillo, facilitando así la labor pedagógica. Este material interactivo agrupa diversas actividades lúdicas de evaluación. Fue procesado en los programas Dreamweaver, Macromedia Flash y Fireworks, y diseñado en formato de página web ejecutado en EDILIM v 3.2. El software fue evaluado con estudiantes de dos instituciones educativas del área de Ciencia Tecnología y Ambiente, ubicados en la ciudad de Iquitos, región Loreto, Perú. Los resultados muestran que los estudiantes de ambas instituciones tuvieron un incremento significativo en la comprensión sobre la conservación y manejo de la especie, a través de las TIC, posibilitando que los estudiantes sean capaces de construir su propio proceso de aprendizaje por medio de la experimentación, innovación, difusión y uso compartido de información y de buenas prácticas.

PALABRAS CLAVE: Arapaima gigas, paiche, TIC, interactivo, innovación.

\section{KNOW AND VALUE OUR AMAZON NATURAL RESOURCES THROUGH INFORMATION, COM M UNICATION AND TECHNOLOGY (ICT): Arapaima gigas}

\begin{abstract}
The lack of means and materials, practical design, production and use of educational resources, based on the needs of students, hamper the improvement of educational quality in many educational institutions of Loreto and the country. Faced this reality is necessary to promote the design, production and use of educational resources, playing in that an important role the Technologies Information and Communication Technology (ICT), which in future will improve the teaching and learning process. In this way was prepared "Giant Amazon: Arapaima gigas paiche" interactive material with the aim of helping to innovate the curriculum and the consolidation of the values $8 \mathrm{f}$ conservation and Amazon resource management at an early age. The technical information was translated into easily language to facilitate the educational work. It contains many interactive play activities for evaluation and was processed in the Dreamweaver, Macromedia Flash and Fireworks programs, designed in a web page format EDILIM executed in v 3.2. The software was tested with students from two educational institutions in the area of Science Technology and Environment, located in the city of Iquitos, Loreto, Peru. The results show that students of both institutions had a significant increase in understanding about the conservation and management of the species, through ICT, enabling the students to be able to build their own learning process through experimentation, innovation, dissemination and sharing of information and best practices.
\end{abstract}

KEYW ORDS: Arapaima gigas, paiche, ICT, interactive, innovation 


\section{INTRODUCCIÓN}

Con la llegada de las tecnologías informáticas, el rol del docente está cambiando desde un enfoque centrado en el profesor - prácticas alrededor del pizarrón y el discurso, basado en clases magistrales hacia una formación centrada principalmente en el alumno, dentro de un entorno interactivo de aprendizaje (UNESCO, 2004). En ese sentido, las Tecnologías de la Información y Comunicación (TIC) juegan un rol importante al proveer a los estudiantes la información y conocimientos necesarios para el proceso de enseñanza-aprendizaje.

Diversos autores plantean la necesidad de integrar las TIC al currículo, de forma que su uso responda a necesidades y demandas educativas (Reparaz et al., 2000; Escudero, 1992; Rodríguez, 1995). Para Dockstader (1999) integrar curricularmente las TIC es utilizarlas eficiente y efectivamente en áreas de contenido general, permitiendo que los estudiantes adquieran habilidades computacionales en formas significativas. Se trata de incorporar las TIC de manera que faciliten el aprendizaje de los alumnos. También se trata de usar el software para que los alumnos aprendan a usar los computadores flexiblemente, con un propósito específico y creativamente. Otro factor clave es la diversificación de contenidos y métodos, lo que promueve la experimentación, la innovación, la difusión y el uso compartido de información y de buenas prácticas.

Es importante destacar que las TIC ofrecen la posibilidad de proporcionar ambientes inteligentes de interacción, promoviendo en los estudiantes el abandono del comportamiento pasivo hacia una actitud activa constante. Por otro lado, estas tecnologías incrementan la posibilidad de que los estudiantes desarrollen sus tareas y tengan iniciativas para tomar "pequeñas" decisiones, para filtrar información, para escoger y seleccionar, así como para utilizar aplicaciones interactivas para su propio aprendizaje, dentro de un marco ético, potenciando el aprendizaje autónomo a lo largo de la vida. (Palomo et al., 2006; ME-DCN, 2009). Todos estos cambios propician nuevas formas de vida, producción y trabajo, lo cual demanda que las instituciones educativas de todos los niveles, y especialmente del nivel primario y secundario, orienten sus propósitos a la formación de individuos creativos, con habilidades para enfrentar los desafíos emergentes de la globalización y participar de forma innovadora en la solución de los problemas sociales y productivos. Para que el uso de las TIC favorezca al proceso enseñanzaaprendizaje es esencial que tanto los futuros docentes, como los docentes en actividad, sepan utilizar estas herramientas que contribuyen a innovar el currículo educativo regional con base en las necesidades de los estudiantes.

El paiche es una especie emblemática del ecosistema amazónico (Araujo \& Goulding, 1997), es considerada una de las mayores especies de peces de agua dulce del mundo, y tiene gran importancia científica, económica, ambiental y cultural, lo que que sustentan la necesidad de impulsar su conservación y desarrollar su potencial como una alternativa productiva para los habitantes de la región amazónica.

Esta especie alcanza hasta $2.5 \mathrm{~m}$ de longitud total y $250 \mathrm{~kg}$ de peso, presenta respiración aérea obligatoria, y posee una excelente calidad de carne (ausencia de espinas intermusculares y bajo contenido en grasas). Ello hace que adquiera elevado valor en el mercado, incentivado también una intensa presión de pesca, con la consecuente reducción y erosión genética de sus poblaciones naturales (Alcántara et al., 2006;). Esto ha obligado a incluirla dentro del Apéndice II del CITES, como una especie amenazada y de comercialización restringida, y dentro de la IUCN en la categoría de especie con datos insuficientes, debido a que la información acerca de sus poblaciones naturales es escasa (IUCN, 2011).

El paiche juega un papel importante en el mantenimiento del equilibrio y en la composición de las comunidades ícticas presentes en los cuerpos de agua (Padilla et al., 2003; Crossa et al.; 2003; García $\&$ Motreuil, 2003). Por estas razones es importante conocerlo, conservarlo, valorarlo y aprovecharlo de manera sostenible. Esto es lo que propone la Resolución Ministerial $\mathrm{N}^{\circ}$ 215-2001-PE, que establece el período comprendido entre los meses de marzo y setiembre como la temporada anual de pesca del "paiche" en los cuerpos de agua públicos del país, a excepción de los de la cuenca hidrográfica del río Putumayo, quedando prohibida la extracción de dicha especie desde el 1 de octubre de cada año hasta el 28 de febrero del año siguiente.

Lo aquí citado muchas veces es ignorado o desconocido por algunos pobladores de la región amazónica, existiendo enormes vacíos de información que conllevan a no valorar este recurso. Por esa razón, se seleccionó esta especie para elaborar el presente material interactivo en el sistema TIC, porque contribuye a la consolidación de los valores de conservación y manejo de los recursos amazónicos a edades tempranas, a través de tecnologías que despiertan un gran interés entre los niños, como son la computación y el internet. 


\section{MATERIAL Y MÉTODOS}

\section{ÁREA DELESTUDIO}

El material educativo fue aplicado en 1500 estudiantes de $1^{\circ}$ al $5^{\circ}$ grado de nivel secundario de dos Instituciones Educativas, la I. E. I. P. S. M "Mariscal Oscar R. Benavides" (MORB) y la I. E. Parroquial "Nuestra Señora de la Salud" (IEPNSS), Iquitos 2010, de la región Loreto, Perú. En ambos casos se promovió el uso de las TIC con temas relacionados con los recursos naturales amazónicos.

\section{METODOLOGÍA}

Consistió básicamente en la recopilación y adecuación de información bibliográfica sobre el paiche Arapaima gigas. La información técnica fue traducida a un lenguaje de fácil comprensión por los niños, con miras a innovar el currículo educativo, de acuerdo con las necesidades de los estudiantes de la región Loreto. Esta información fue procesada en los programas Dreamweaver, Macromedia Flash y Fireworks, el que fue diseñado en formato de página web. El contenido considera, principalmente, las siguientes secciones: historia, descripción, taxonomía, nombres populares, morfología, hábitat, distribución geográfica, reproducción, conservación, información nutricional, gastronomía, una sección interactiva (¿sabías qué?) y actividades.

Para la evaluación se utilizó el programa EDILIM v 3.26 (Macías, 2006) que consta de diversas actividades como: (i) rompecabezas, que plantean la reconstrucción de una información desordenada, la que puede ser gráfica, textual o sonora, o combinar aspectos gráficos y auditivos al mismo tiempo; (ii) asociaciones, que pretenden ayudar al usuario a descubrir las relaciones existentes entre dos conjuntos de información; (iii) sopas de letras, con variantes interactivas de los conocidos pasatiempos de palabras escondidas, como completar párrafos, responder preguntas, clasificar textos, etiquetas, respuesta múltiple e identificar imágenes.

Antes de aplicar el material interactivo se realizó una encuesta a un $30 \%$ de estudiantes de las dos instituciones educativas, con la finalidad de obtener información sobre las potencialidades y limitaciones de los estudiantes respecto al uso de las TIC en el proceso de enseñanza - aprendizaje, y sobre el conocimiento de la especie.

\section{RESULTADOS Y DISCUSIÓN}

\section{GRADO DE CONOCIM IENTOS A PRIORI DEL MATERIAL EDUCATIVO}

El análisis de las encuentras aplicadas a priori muestra que un $60 \%$ de los estudiantes de la I. E. I. P. S. M. "Mariscal Oscar R. Benavides" no hace uso de las TIC, pese a contar con una Aula de Innovación Pedagógica (Sala de Cómputo). En cuanto que en la I. E. Parroquial "Nuestra Señora de la Salud" un $80 \%$ de los estudiantes tiene buen conocimiento sobre las TIC, y hace uso frecuente de los recursos informáticos (Tabla 1).

Con respecto al conocimiento de la especie los resultados de la encuesta indican que el 75\% de los estudiantes de la I. E. I. P. S. M. "Mariscal Oscar R. Benavides" tiene información sobre el paiche. Esto es debido, en gran parte, a que cuentan con un estanque para la crianza del Arapaima gigas dentro del Área "Educación para el trabajo", y con asistencia técnica de los especialistas de Educación ambiental y del Programa de Investigación en el Uso y Conservación del Agua y sus Recursos (AQUAREC), del Instituto de Investigaciones de la Amazonía Peruana (IIAP). En contraste, sólo un 50\% de estudiantes de la I. E. Parroquial "Nuestra Señora de la Salud" tiene información sobre la especie. Esto muestra que los sistemas informáticos son usados preferencialmente para la obtención de otro tipo de información y el acceso a redes sociales (Tabla 2 ).

\section{GRADO DEACEPTACION DEL M ATERIAL INTERACTIVO}

Conociendo el grado de información de estos dos grupos de estudiantes, se aplicó el material interactivo "El gigante del Amazonas paiche Arapaima gigas", obteniéndose un $65 \%$ de aceptación por parte de los estudiantes de la I. E. I. P. S. M. "Mariscal Oscar R. Benavides" y un $75 \%$ en los estudiantes de la I. E. Parroquial "Nuestra Señora de la Salud". Pensamos que el elevado grado de aceptación se debe, principalmente, a que el material es atractivo y lúdico, además de poseer multimodalidad de lenguaje e interactividad. Pese a que un $35 \%$ de estudiantes de la I. E. I. P. S. M. "Mariscal Oscar R. Benavides" desconocía o estaba poco familiarizado con el uso de las TIC, el grado de aceptación fue de sólo 10\% menos que del otro centro educativo. Una de las razones de esta inseguridad inicial se debe a la diferencia en los estratos económicos de los dos grupos analizados, que muchas veces no les permite tener acceso a estas tecnologías. A esto se suma, además, la falta de interés de los docentes con respecto al uso de los recursos educativos relacionados a la informática.

\section{GRADO DE CONOCIMIENTO A POSTERIORI DE LA ESPECIE}

Los resultados muestran que los estudiantes de ambas instituciones tuvieron un incremento en sus conocimientos sobre el paiche, despertándose 
sentimientos de valoración acerca de la especie. Sin embargo, los estudiantes de la I. E. I. P. S. M. "Mariscal Oscar R. Benavides" mostraron mayor interés en la función que cumple la especie en los ecosistemas acuáticos y su potencial económico como una alternativa productiva.

Los resultados muestran que las TIC pueden ser aplicadas en diferentes áreas curriculares, facilitando la labor pedagógica, al ser un material sencillo y dinámico. Por otro lado, nos permiten involucrarnos en el cumplimiento de las demandas educativas que plantean el mundo moderno y la globalización, los avances de la ciencia y la tecnología, la comprensión del medio natural y su diversidad, la promoción de la seguridad alimentaria y la nutrición, así como el desarrollo de una conciencia ambiental orientada a la gestión de riesgos y al uso racional de los recursos naturales. Todos estos aspectos están contemplados en el marco de una moderna ciudadanía, como propósitos de la Educación y las Políticas de Estado del Perú al 2021(ME-PEN 2010).

Desde esta perspectiva, es importante que los planes y programas de estudio se actualicen de acuerdo con las demandas de la sociedad actual, lo cual implica rediseñarlos bajo el enfoque de un modelo educativo por competencias centradas en el aprendizaje interactivo, a partir de aspectos sociales y ambientales, vinculados al cuidado de la salud y su relación con el desarrollo tecnológico. Todo lo mencionado contribuirá a tener un mayor conocimiento y valoración de la especie, por su importancia en los ecosistemas amazónicos, así como promoverá la formación pertinente del estudiante frente a los cambios acelerados del contexto global.

Como en todo proceso de enseñanza-aprendizaje, consideramos que la integración de las TIC al currículo educativo regional es muy significativa en las instituciones educativas de la región Loreto, Perú, ya que provee a los estudiantes información que permite enriquecer sus conocimientos y los capacita para construir su propio proceso de aprendizaje, al mismo tiempo que promueve la experimentación, la innovación, la difusión y el uso compartido de información y de buenas prácticas. Pero consideramos que este proceso debe ser gradual debido a que depende del comportamiento de muchas variables relacionadas con cuatro factores: 1) recursos tecnológicos propiamente dichos, hardware y software; 2) filosofía pedagógica y competencia tecnológica de los educadores; 3) disponibilidad y correcta utilización de los contenidos digitales apropiados; y 4) apoyo administrativo, pedagógico y técnico que ofrece la institución educativa.

Tabla 1. Resumen de la encuesta realizada al 30\% de la población analizada de los centros educativos: E. I. P. S. M . "M ariscal Oscar R. Benavides" y I. E. Parroquial "Nuestra Señora de la Salud"

\begin{tabular}{ccccc}
\hline $\begin{array}{c}\text { INSTITUCIONES } \\
\text { EDUCATIVAS }\end{array}$ & POBLACIÓN & ENCUESTADOS & $\begin{array}{c}\text { NO HACEN USO } \\
\text { TIC"S }\end{array}$ & $\begin{array}{c}\text { CONOCIMIENTO } \\
\text { DE LA ESPECIE }\end{array}$ \\
\hline MORB & 750 & 225 & $135(60 \%)$ & $169(75 \%)$ \\
IEPNSS & 750 & 225 & $180(80 \%)$ & $112(49 \%)$ \\
\hline
\end{tabular}

Fuente: Encuesta aplicada a estudiantes 2010.

Tabla 2. Aceptación del material educativo por los estudiantes de los centros educativos: E. I. P. S. M. "M ariscal Oscar R. Benavides" y I. E. Parroquial "Nuestra Señora de la Salud"

\begin{tabular}{ccc}
\hline $\begin{array}{c}\text { INSTITUCIONES } \\
\text { EDUCATIVAS }\end{array}$ & POBLACIÓN & ACEPTACIÓN \\
\hline MORB & 750 & $488(65 \%)$ \\
IEPNSS & 750 & $563(75 \%)$ \\
\hline
\end{tabular}

Fuente: Aceptación del material aplicado a estudiantes 2010. 


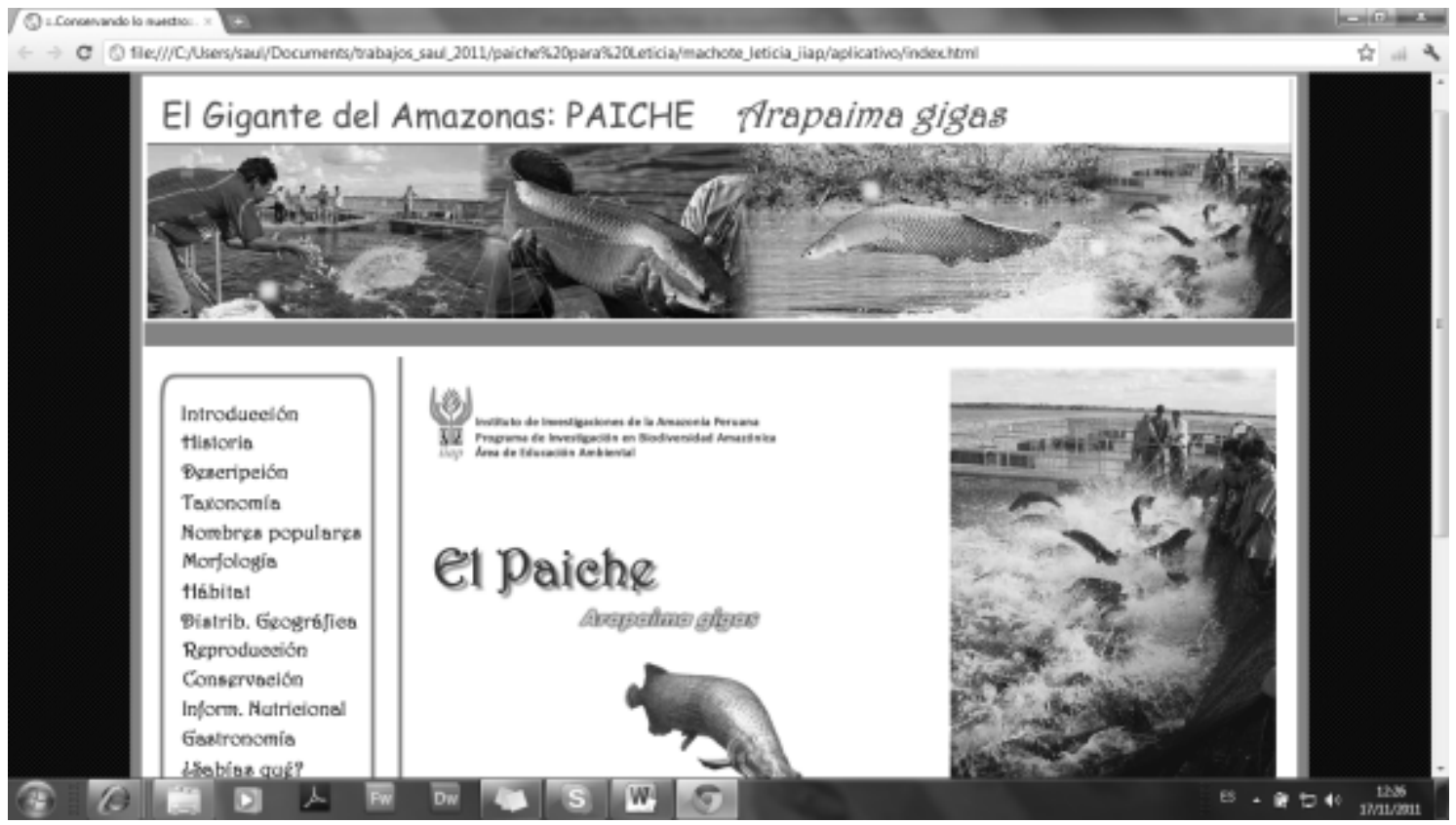

Figura 1. Imagen mostrando la página principal del material interactivo "El gigante del Amazonas paiche Arapaima gigas"

\section{AGRADECIMIENTOS}

Los autores agradecen a M.Sc. José Álvarez Alonso y a Dra. Carmen García-Dávila por la revisión del manuscrito de este trabajo. Al Dr. Fred Chu-Koo por el invalorable apoyo prestado durante el desarrollo de este estudio.

\section{BIB LIOGRAFÍA CITADA}

Alcántara, F.; Guerra, H.1992. Cultivo de paiche, Arapaima gigas, utilizando bujurqui, cichlassoma bimaculatum, como presa. Instituto de Investigaciones de la Amazonía Peruana, Folia Amazónica, 4(1) 12 pp.

Alcántara, F.; Wust, W.; Tello, S.; Rebaza, M.; Del Castillo D. 2006. Paiche El Gigante del Amazonas. Instituto de Investigaciones de la Amazonía Peruana, Wust ediciones, 69 pp.

Araújo-Lima, C.; Gouding, M. 1997. Os frutos do tambaqui: ecologia, conservação e cultivo na Amazônia. Tefé, AM. Sociedade Civil Mamirauá, Brasília-CNPq-MCT. 186 pp.

Crossa, M.; Rocha, W.; Pinto-Sá, E. 2003. Investigación participativa. Una experiencia promisora para el subsidio de programas de manejo del piraruçu (Arapaima gigas Cuvier) en el bajo Amazonas. Libro de resúmenes del
"Seminario Taller Internacional de Manejo de Paiche o Pirarucu". IIAP. Iquitos - Perú. 67-81pp.

Dockstader, J. 1999. Teachers of the 21scentury know the what, why, and how of technology integration. T.H.E. Journal, 73-74 pp.

Del Águila, J. 2002. Plan de Manejo de paiche en las cochas de Punga. Junglevagt For Amazonas WWF-AIF/DK, Programa Integral de Desarrollo y Conservación Pacaya Samiria. Iquitos, Perú. 115 pp.

Escudero, J. 1992. La integración escolar de las nuevas tecnologías de la información. Infodidac, Revista de Informática y Didáctica : 21,11-24 pp.

García, A.; Montreuil, V.; 2003. La veda como un instrumento de manejo del paiche en Loreto. Libro de resúmenes del "Seminario Taller Internacional de Manejo de Paiche o Pirarucu". IIAP. Iquitos Perú. 83 - 89.

Macías F. 2006. Software Educativo EdiLim V.3.26. http://www.educalim.com

Ministerio de Educación. 2010. Proyecto Educativo Nacional al 2021 "La Educación que queremos para el Perú". Lima-Perú. 150 pp

Ministerio de Educación. 2009. Diseño Curricular Nacional de la Educación Básica Regular Segunda Edición. Lima- Perú. 478 pp 
Ministerio de Pesquería. 2001. RESOLUCION MINISTERIAL N $\mathrm{N}^{\circ}$ 215-2001-PE. Establecen temporada anual de pesca del recurso "paiche" en cuerpos de agua públicos del país.

Padilla, P.; Ismiño. R.; Alcantara, F.; Tello, S. 2003. Producción y Manejo de Alevinos de Paiche en Ambientes Controlados. Libro de resúmenes del "Seminario Taller Internacional de Manejo de Paiche o Pirarucu". IIAP. Iquitos - Perú. 125 $141 \mathrm{pp}$

Palomo, R.; Ruiz, J.; Sanchez, J. 2006. Las TIC como agentes de Innovación Educativa. España. Capitulo II, $29 \mathrm{pp}$

Pronaturaleza. 2004 - 2008. Plan de manejo de Arapaima gigas "paiche" en la cocha el Dorado, cuenca Yanayacu Pucate - Reserva Nacional Pacaya Samiria,. Loreto-Perú. 88 pp
Reparaz, Ch.; Sobrino. A.; Mir, J. 2000. Integración curricular de las nuevas tecnologías. Barcelona: Editorial Ariel S.A. Barcelona. 169 pp

Rodríguez, J.L. 1995. Tecnología educativa: Nuevas Tecnologías Aplicadas a la Educación. Alcoy, Marfil. 187-213 pp

World Conservation Monitoring Centre 1996. Arapaima gigas. In: IUCN 2011. IUCN Red List of Threatened Species. Versión 2011.2

UNESCO. 2004. Las tecnologías de la información y la comunicación en la formación docente. Uruguay: Ediciones Trilce. 244 pp 